\title{
Current Status of Neurointerventional Activities in Korea
}

\author{
Yong-Moon Lee, RT, Seon Moon Hwang, RT, Eun Hye Kim, RN, Dong-geun Lee, MD, \\ Jae Ho Shim, MD, Dae Chul Suh, MD
}

Neurointervention which deals with neurovascular disease in brain, head-and-neck and spines are one of the rapidly evolving medical fields. Several indices representing neurointerventional activities are still increasing year-by-year in Korea. We review current trend in major neurovascular diseases requiring usage of specific medical devices and some issues related to administrative process of the government approval.

Key Words : Neurointervention; Angiomachine; Devices

The population of the South Korea was about $50,767,000$ in 2012. The main cause of death in Korea was cancer, cerebrovascular disease, cardiac disease, suicide, and diabetes in the order of frequency in 2011 [1]. Although average life expectancy of Korean was 81 years in 2011, healthy lifespan still remains low (Fig. 1) [2]. There are four serious illnesses (cancer, cerebrovascular and cardiovascular illnesses, and rare hard-to-treat disease) which can be covered by insurance more than $95 \%$ in contrast to other disease $80 \%$. That means the deductable (the amount patients pay for) stands at $20 \%$ for general illness, at $5 \%$ for the cancer, cerebrovascular and cardiovascular illnesses (only for surgery and recanalization), and at $10 \%$ for rare, hard-to-treat

All authors: Department of Radiology and Research Institute of Radiology, University of Ulsan, College of Medicine, Asan Medical Center, Seoul, Korea

Received August 3, 2013; accepted after revision August 9, 2013.

Correspondence to: Dae Chul Suh, MD, Department of Radiology, University of Ulsan, College of Medicine, Asan Medical Center, 88, Olympic-ro 43-gil, Songpa-gu, Seoul 138-736, Korea.

Tel. 82.2.3010.4366 Fax. 82.2.476.0090

E-mail: dcsuh@amc.seoul.kr

This is an Open Access article distributed under the terms of the Creative Commons Attribution Non-Commercial License (http://creativecommons.org/licenses/by-nc/3.0) which permits unrestricted non-commercial use, distribution, and reproduction in any medium, provided the original work is properly cited. diseases [3].

Although expertise requires long period of medical training [4], neurointervention is one of the major medical field which progresses rapidly and can profoundly induce development of new devices or materials so that the neurointerventional procedure can affect health index in the era of longevity [5]. Therefore, we present current status of facilities, devices and trends of procedures related to this new medical field.

\section{Number of Angiomachines}

The number of angiomachines increased 5\% in 2012 and 4\% in 2013 nationwide due to continuous new installation (Fig. 2). Installation number of angiomachine in each hospital depends on the scale of the hospital (Fig. 3). Most angiomachines were installed in the tertiary or the general hospital which has more than 100 beds. There were overall 379 angiomachines; single 255 and biplane 124 in 2011.

\section{Neurointerventional Devices and Materials}

The current insurance coverage and cost of neurointerventional devices and materials are shown in Fig.4. Moma (Invatec, Roncadelle, Italy), Solitaire (EV3 Inc., 
CA, USA), drug eluting stent, and carotid protection device are not covered by insurance yet. Although stent-retriever like Solitaire can be very effective in clot removal in patients with acute stroke, there is no way to use it because generation of cost by using it is not

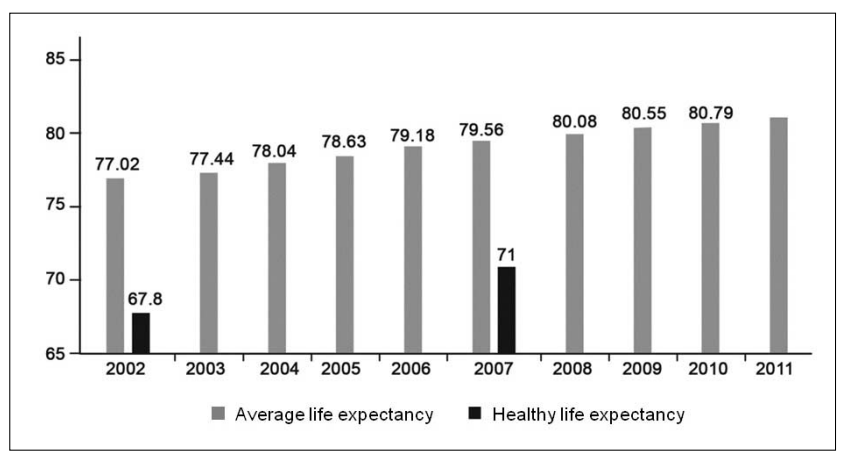

Fig. 1. Annual average and healthy life spans of Koreans.

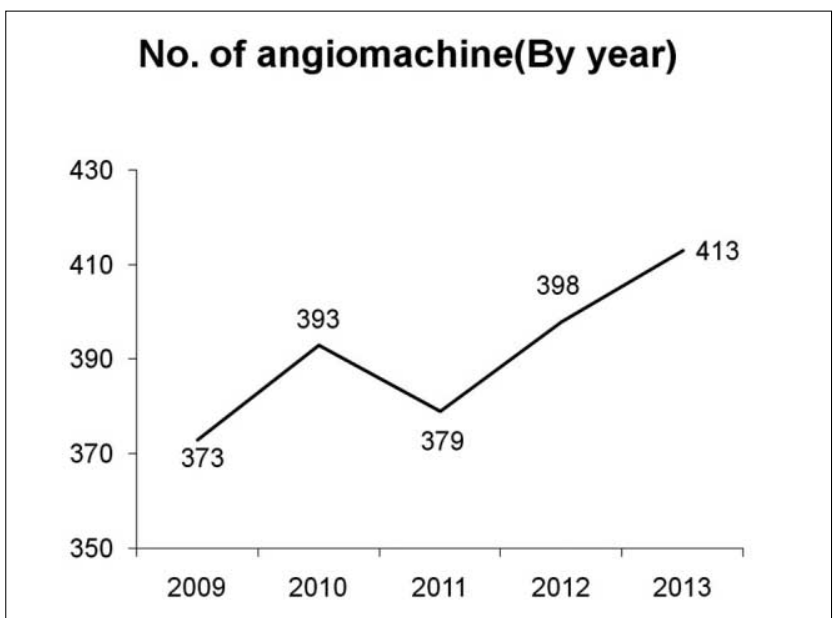

Fig. 2. Annual change of angiomachine in Korea nationwide. The number in 2013 was estimated by doubling the number of angiomachin in the first half year.

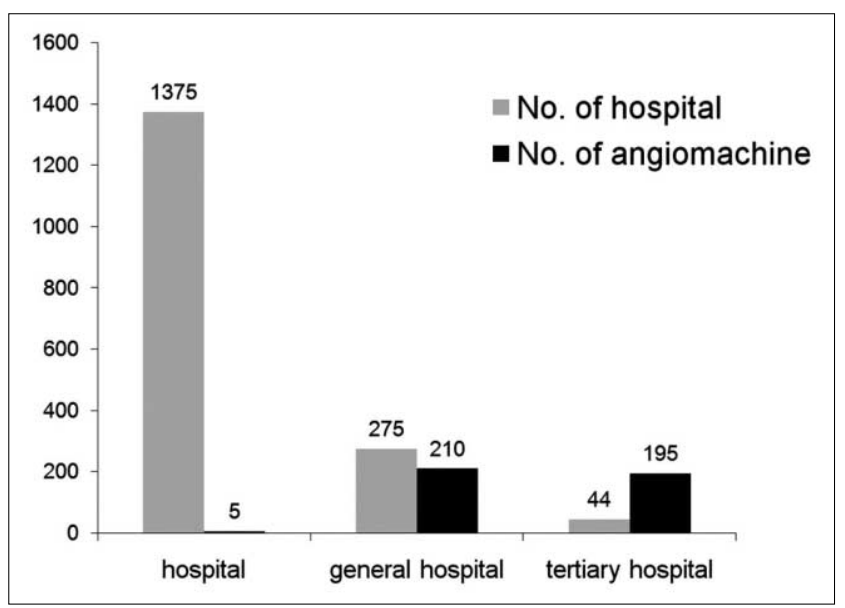

Fig. 3. Number of angiomachine according to the hospital scale. allowed unless the device is allowed to be used by the Korean FDA and the insurance coverage is assessed by Health Insurance Review \& Assessment Service. Nowadays the process takes almost two years if the medical methodology using the device is recognized as a new technology.

\section{Trends of Case Number of Major Procedures}

\section{Aneurysms:}

There is increasing tendency of case number yearly (Fig. 5). The reason of the increase can be explained by more detection of unruptured aneurysm rather than due to increased ruptured aneurysm. Although case number of clipping is still more than that of coiling, total clipping number decreased during the last two years in contrast to the continuous increase of coiling number.

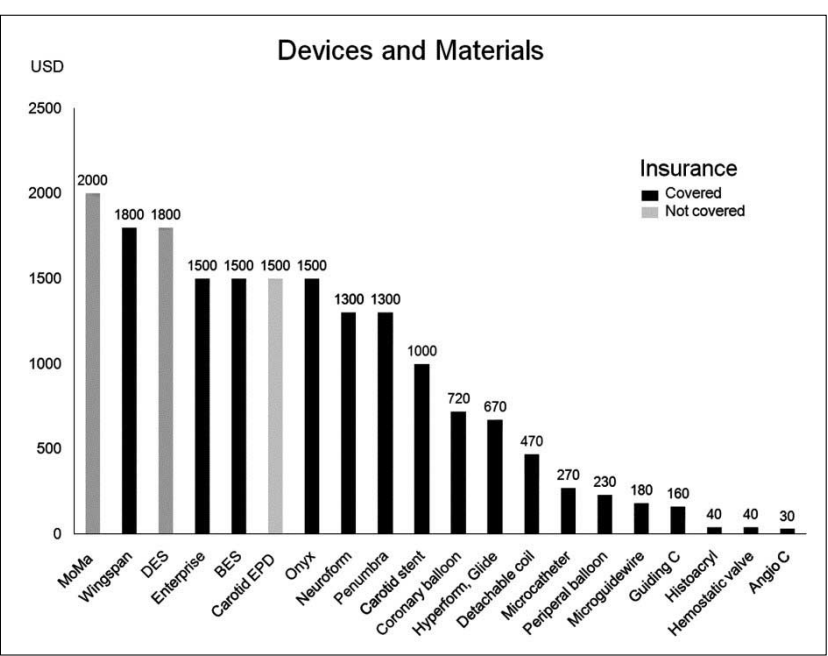

Fig. 4. Current cost and insurance coverage of devices and materials. Gray color bars including histoacryl means those not covered by insurance.

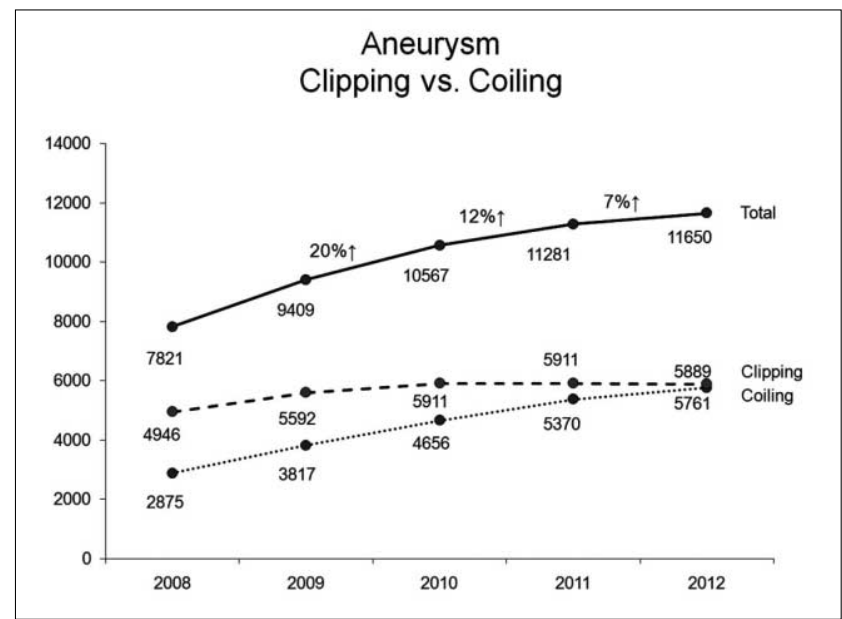

Fig. 5. Annual trends of procedural numbers; clipping vs. coiling. 


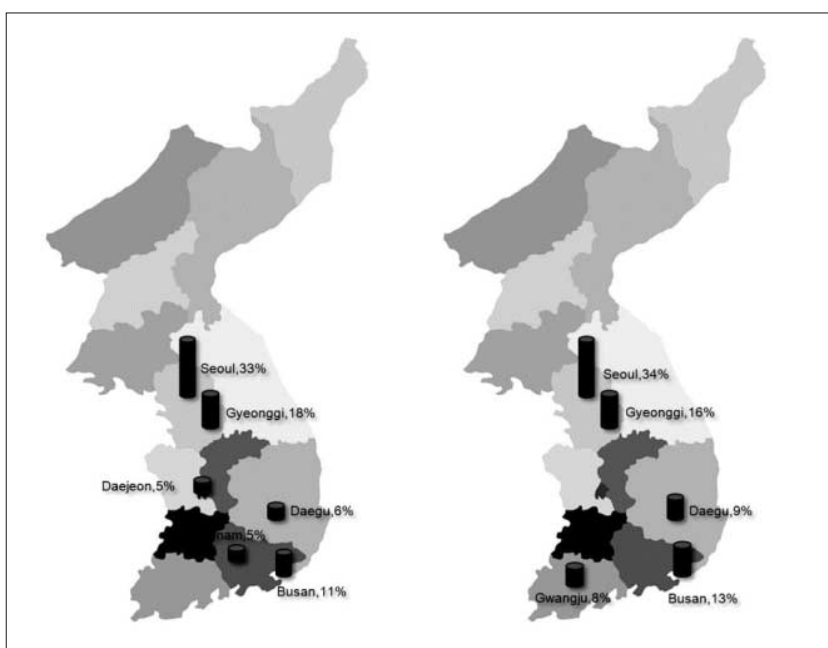

Fig. 6. Geographic distribution of the number of procedures; coiling (A) vs. clipping (B).

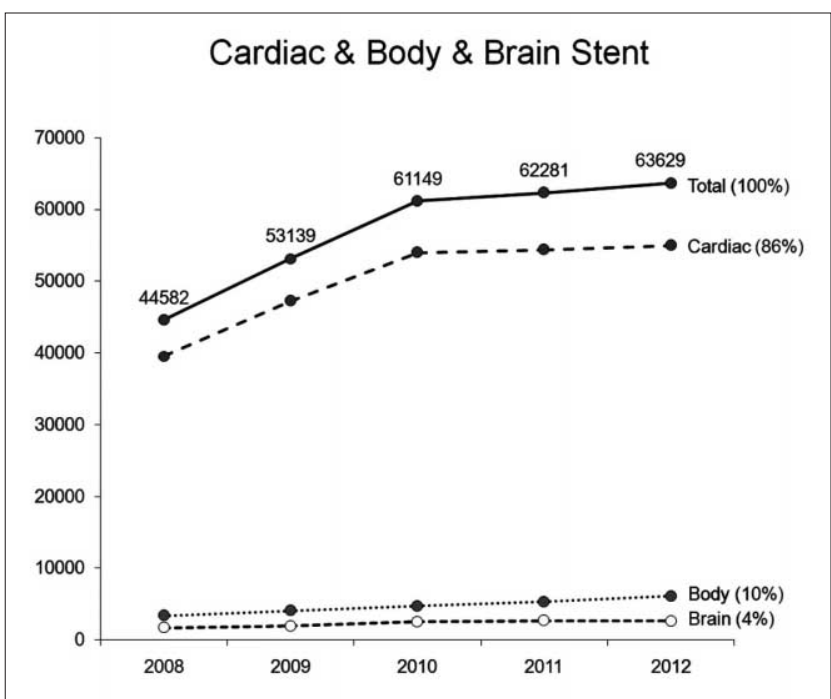

Fig. 7. Annual trends of case numbers of cardiac, body and brain stentings.

Therefore, the increasing trend of total number can be explained by increase of coiling number. It is expected that the number of coiling surpass the number of clipping in 2013.

The most procedures were performed in large cities (Fig. 6). The case number of Seoul and Gyeonggi consisted of almost $50 \%$ of the total number. Such trend is similar in both coiling and clipping. However, clipping prevailed in some region as in Gwangju while coiling prevailed in Daejeon and Gyeongnam areas.

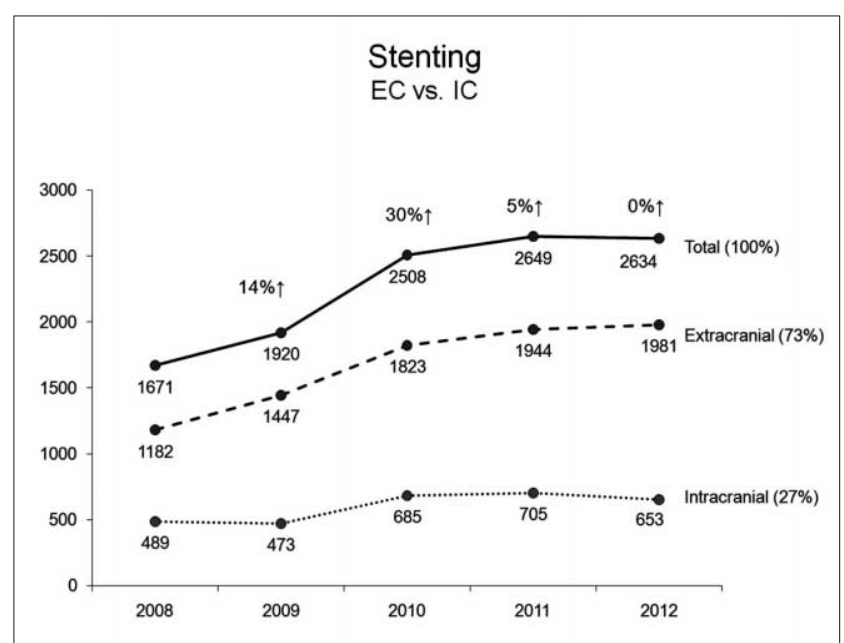

Fig. 8. Annual trends of case numbers of brain stentings; extracranial vs. intracranial.

\section{Stenting Procedures}

There is increasing tendency of overall stenting procedures in Korea. Cardiac stenting consisted of $86 \%$, body $10 \%$ and brain (Neuro) only $4 \%$ of the total cases (Fig. 7). Regarding neuro-stenting, extracranial procedures consisted of $73 \%$ and intracranial procedures $27 \%$ (Fig. 8). Overall number of stenting procedures is recently stationary because the number of extracranial procedures increases while the number of intracranial procedures decreases.

In conclusion, the neurointerventional activities are increasing in every aspect of data including installation number of angiomachine and case number of procedures. In contrast to the needs and increasing tendency of the neurointerventional procedures, administrative process of the government takes rather long time in recognizing new medical technology and assessing effectiveness of the device and materials.

\section{References}

1. Cause of death statistics in 2011, Statistics Korea

2. Trend of life span, http://www.index.go.kr/, Statistics Korea

3. Korean Statistical Information Service, http://kosis.kr/

4. Zhao LB, Miyachi S, Shi HB, Suh DC. Comparison of medical education and requirements for training in the interventional neuroradiology in China, Japan and Korea. Neurointervention 2013;8:3-8

5. Ryu CW, Hwang SK, Jung HD, Park YS, Park MH, Kim HJ. The present status of neurointerventional angiographic systems and workers in Korea. Neurointervention 2009;4:116-124 